\title{
The rich afterimage illusions under head-image tilting and the internal visual space model
}

\author{
Gary M Zenkin* \\ Institute for Information Transmission Problems, (Kharkevich Institute) of Russian Academy of Sciences, Moscow, Russian Federation
}

\begin{abstract}
Some features of the illusions arising from the movement of the observer's eyes and/or head under the background of the positive afterimage of the entire visual scene (rich afterimage-rA) when the head and/or afterimage are tilted are described.

Under these conditions, there are the violations of the visual direction constancy and stability of the visual field. This happens also under the constant position of the eyes in orbits when changing the fixation point by the head movements. The illusion of violation of the visual vertical on the background of an inclined $\mathrm{rA}$ is also described.

The features of the so-called "residual illusion" are described in detail. In this phase all phenomena that have arisen against the background of rA persist for tens of seconds in total darkness after the rA is completely attenuated. This also applies to distorted perception of the visual vertical.

Based on the analysis of the rA illusions and peculiar properties of the rich afterimage itself, it is proposed to consider the hypothesis, suggesting the complete nonparticipation of the efferent oculomotor subsystem signals in the mechanisms of the visual field stability and the gaze direction constancy. The hypothesis suggests that the structurally rich visual scene allows the visual system to form a stable visual field and to calculate the observer's gaze direction only based on the signals within the visual flow. The hypothesis allows to coherently interpret the main phenomena have been observed in the experiments.
\end{abstract}

\section{Introduction}

In the experiments described in previous works it has been shown that it is useful to distinguish two types of visual afterimages-the afterimage of the entire visual scene-the "rich" afterimage $(\mathrm{rA})$ and the afterimage of a separate "object"-the "poor" afterimage (pA) [1-3].

It has been found that against the background of $\mathrm{rA}$ (as opposed to poor one) eye movements lead to disturbance of the visual space stability and visual direction constancy. This was expressed in the fact that any eye movement when changing eye position by a saccade or by tracking the moving point does not affect the state of $\mathrm{rA}$ (visible position and size), but leads to an apparent shift of actually stationary luminous points-the markers of real space (MRS) and even change their apparent size.

At the same time, it turns out that in these conditions a false interpretation of the observer's gaze direction also occurs, and this causes an obvious contradiction between the direction of one's own gaze perceived by the observer and the eye positions in orbits. These phenomena were called "the rich afterimage illusion".

Since the observed binocularly $\mathrm{rA}$ appeared in the perception of the observer as a real three-dimensional view of the visual scene, the mechanism of binocular stereo synthesis was recognized as the "culprit" of the illusion [2]. Namely, in conditions when two images of the visual scene are "frozen" on the retinas, the stereopsis fusion mechanisms can create a 3-D undiplopien image of the scene after changing of the eye position only by "returning it to its previous position". Such a "return" leads to an apparent shift of luminous points as markers of real space (MRS).
But in the new experiments, set in wider experimental conditions, it has been found that the illusion in its main features is observed monocular-under monocular rA [3]. Based on these experiences, it have been also concluded that this illusion can be interpreted as the result of a 3-D projective transformation of the coordinate grid of the 3-D internal model of the visual space (IVS), is presented in visual perception as a current actual visual field.

In particular, when changing the fixation in the frontal plane horizontally on the background of a rA, the transformation of the inner visual space (IVS) can be represented as a rotation of the coordinate grid of the 3-D IVS around a vertical axis located in some center of eye rotation [3]. When changing the fixation point in depth, there was also a scale transformation of the IVS grid, which manifested itself in apparent changing of the MRS' size and their location in depth.

The mismatch of IVS with the internal model of the surrounding space (IMSS), leads in our experiments to corresponding disturbances in the observer's motor orientation in real space [1]. And it leads also to the very fact that the observer interprets this view as an illusion.

${ }^{*}$ Correspondence to: Gary M Zenkin, Institute for Information Transmission Problems, (Kharkevich Institute) of Russian Academy of Sciences, Moscow, Russian Federation, E-mail: imz@paragon.ru

Key words: rich afterimage illusion, eye movements, visual field stability, gaze direction, spatial vision, eye position, residual illusion, head tilt

Received: August 01, 2019; Accepted: August 19, 2019; Published: August 23 , 2019 
Note that none of the phenomenon of illusion is observed in the presence of the $\mathrm{pA}$, which in these conditions behaves itself in according with the Emmert's law.

At the same time under tilting the head to the shoulder, the afterimages of both types behave themselves in a similar way-they turn towards the tilt of the head. And the luminous points-MRS, as in normal observation conditions, remain stationary. While changing the fixation point by the horizontal head rotations the rA illusion is fully manifested [3].

In the present work, the $\mathrm{rA}$ illusions were studied in more detail under the horizontal or vertical head rotations when the head and/or rich afterimage were also tilted. In addition, the observed earlier phase of "residual illusion" has been described in more detail [3].

\section{Methods}

As in the early experiments the rA was created by a short-term ( $10 \mathrm{~ms}$ ) flash of two studio photo lamps Grifon-300, which covered the entire experimental room.

A positive afterimage arising under dark adaptation of a subject undergoes several stages, the description of which will be given later. The rA of entire visual scene is monochromatic gray-bluish, volumetric and makes a strong impression on the subjects. Usually naive (inexperienced) subject has been convinced for some time that he is observing a real environment illuminated by a slowly fading blue light. The phase duration of a well-structured $\mathrm{rA}$ under conditions of optimal dark adaptation is 7-12 seconds.

In the experimental room with contrastingly decorated walls there were some pieces of furniture in front of the subject at different distances. And at distances from $40 \mathrm{~cm}$ to $1.5 \mathrm{~m}$ there were several LEDs glowing with red light that contrasted well with the bluish background of rA. In the experiments, these points act as reference markers of real space (MRS).

Being in complete darkness, the subject observed binocularly or monocularly the rA arising after the flash. At the specified moment, the subject performed one or several actions only with the eyes or also with the head (for example, tracking a moving point, changing the fixation point in one of the indicated directions by saccade etc).

At the same time, he focused on apparent changes in the spatial relationship between the "real space", the afterimage and the luminous points-MRS. Sometimes a subject gave a report of the apparent direction of his own gaze, and sometimes of the apparent position of the head.

In the main experiments, two autonomous blocks or rows of the luminous points were used. Each point, according to the instructions, could be the initial or final fixation point or remain a passive MRS, whose "behavior" could tell about the nature of the transformation of the visual field or, more precisely speaking, the IVS. It was possible with the wide set of MRS to form their 2-3 dimensional configurations, which, as has been shown in previous experiments made it possible to identify quite subtle and somewhat paradoxical details of the observed transformations [3]. Some details of the technique will be given further when describing the results of experiments.

The verbal report of the subject about the observed effects, as well as the online dialogue with the experimenter, was recorded on a sound recorder for further analysis. The illustrations given here in the article to the descriptions of the observed effects were monitored by the subjects themselves for good compliance with the observed phenomena.
In experiments with an inclination of the head and/or afterimage, there are 4 simple combinations of the mutual state, which can be represented in a code table, in which the state of the head-afterimage pair looks like this: $00,10,01,11$, where 0 is the direct position of the head or image, 1-their inclined position. All 4 cases were used in the experiments.

The decoding of these states is given below:

00-the vertical position of the head and the direct orientation of the afterimage;

10-the subject tilts his head to the shoulder before the flash and holds it in this position until the complete attenuation of the rA;

01- the subject tilts his head before the flash, and after returns it to its normal position (sometimes it happens in different phases of the rA development);

11-the subject tilts his head after the flash and keeps it in this position all the time specified by the instructions.

Experienced adult subjects with a normal state of binocular vision took part in the experiments. In different series of experiments, their number varied from 5 to 8 . All variants of the rA illusion for position 00 were well illustrated in an early paper [3].In current experiments, illusion and other effects were studied in more detail mainly under the head-afterimage states 10,01 , and 11 .

\section{Results}

Since this paper will focus mainly on the inclined position of the head and/or rA, here we will illustrate the rA illusion with two relevant examples.

\section{The illusion of a "smooth vertical shift of the IVS" while tracking a moving point (code situation 01)}

The experiment with tracking a moving fixation point was usually carried out at the initial stage of work with each subject, because it did not require any active actions from the last. This allowed the observer to acquire the skill of strict fixation and concentration of attention on a faintly glowing point against the background of a bright impressive afterimage, occupying the entire visual field and, of course, exciting the attention of the subject. In the initial stages, the afterimage often did not allow for a subject to control oneself and focus on the effects associated with such minor stimuli as the reference points-the MRS (Figure 1).

Figure $1 \mathrm{~b}$ shows how the illusion of movement looks like. The subject saw the moving point as stationary, while all the other stationary ones began to move in the opposite direction. As a result, the entire configuration of the points moved from bottom to top and was located near the ceiling of the virtual oblique experimental room.

At the same time, each subject also noted a clear change in the slope of the straightened block of the points $b$ (1-3), as shown in Figure 1b. According to the evaluation of the subjects, the illusory angle of this block inclination was about $20^{\circ}$. This corresponded to the turning angle of the subject's gaze when tracking the a 2 point moved from top to bottom.

Along with these effects, the most subjects reported a subjective impression that the direction of their gaze in space has not changed. The entire path of movement of a2 point in the visual field from top to bottom was $20^{\circ}-25^{\circ}$ and the real movement of the a 2 point lasted 5-8 seconds. 
The oblique orientation of rA did not explicitly affect the features of the illusion, if we compare it with the illusion in the normal (00) head-image position. It should also be noted that a similar illusion arises when the fixation point changes, for example, from a 3 to a1 by saccade.

It should also be specifically noted that this illusion in all fixed in the experiments parameters is also manifested itself under monocular observation (Figure 1b).

\section{The illusion of a "smooth shift of IVS" in depth when tracking a point approaching the observer (situation 10)}

Figure $2 \mathrm{~b}$ shows what the observer's visual field looked like in the final phase of the illusion. All the time of illusion, the subject sees the moving point $\mathrm{b} 2$ as a stationary one, while all the other stationary points MRS moving in the direction from the subject. At the same time, the attentive experienced subject noted a clear change in the slope of the straightened block of points a (1-3).

The apparent shift of the entire configuration of the MRS also has a horizontal component, since the axis of the aligned block b (1-3) was oriented at some angle to the observer's gaze axis.

The entire path of the point's movement in depth was $30-50 \%$ of the distance along the gaze axis, and the real movement of the $b 2$ point lasted for 5-8 seconds. It should be noted that a similar illusion arises when the fixation point changed abruptly e.g. from b3 to b1 with the saccade.
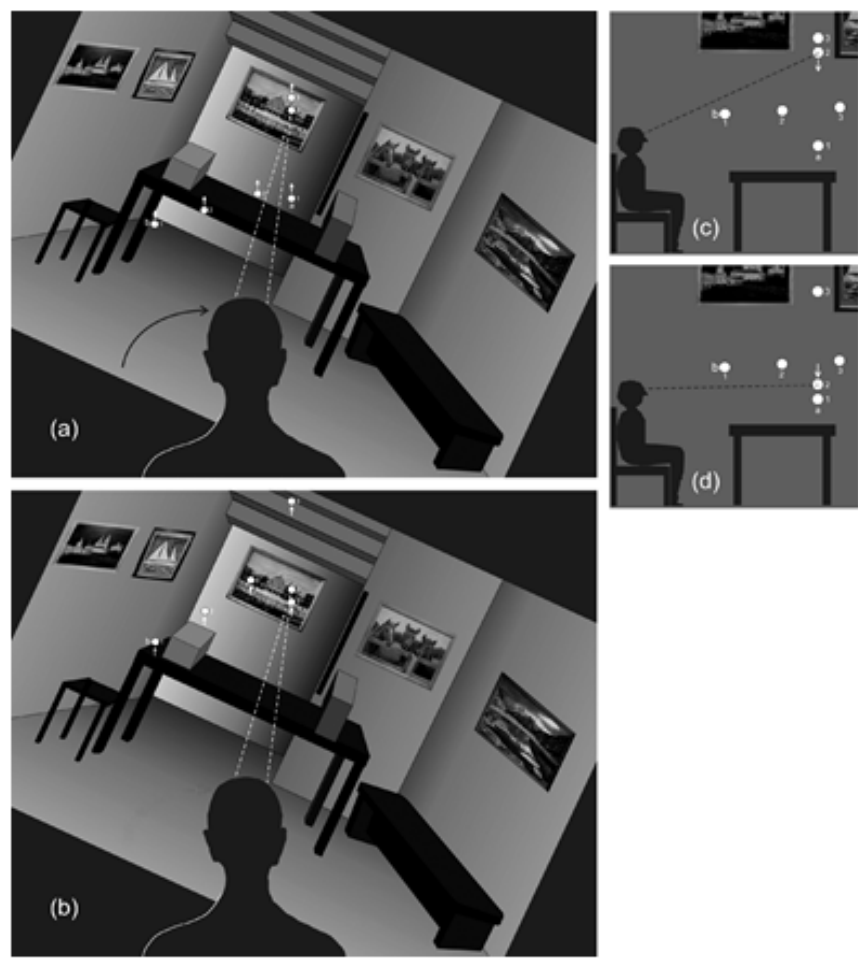

Figure 1. The illusion of "shifting the internal visual space (IVS)" along the vertical axis when tracking a smoothly moving point. (a) The visual field of the subject in the optimal development phase of rA. The subject tracks the point a2 smoothly moving from top to bottom against the background of the entire visual scene afterimage (rA); (b) the apparent movement of an objectively stationary configuration of MRS in the opposite direction with apparent immobility of the actually moving point a2; (c), (d) the actual location of the MRS in the initial (c) and final (d) tracking phases of the moving point (side view)
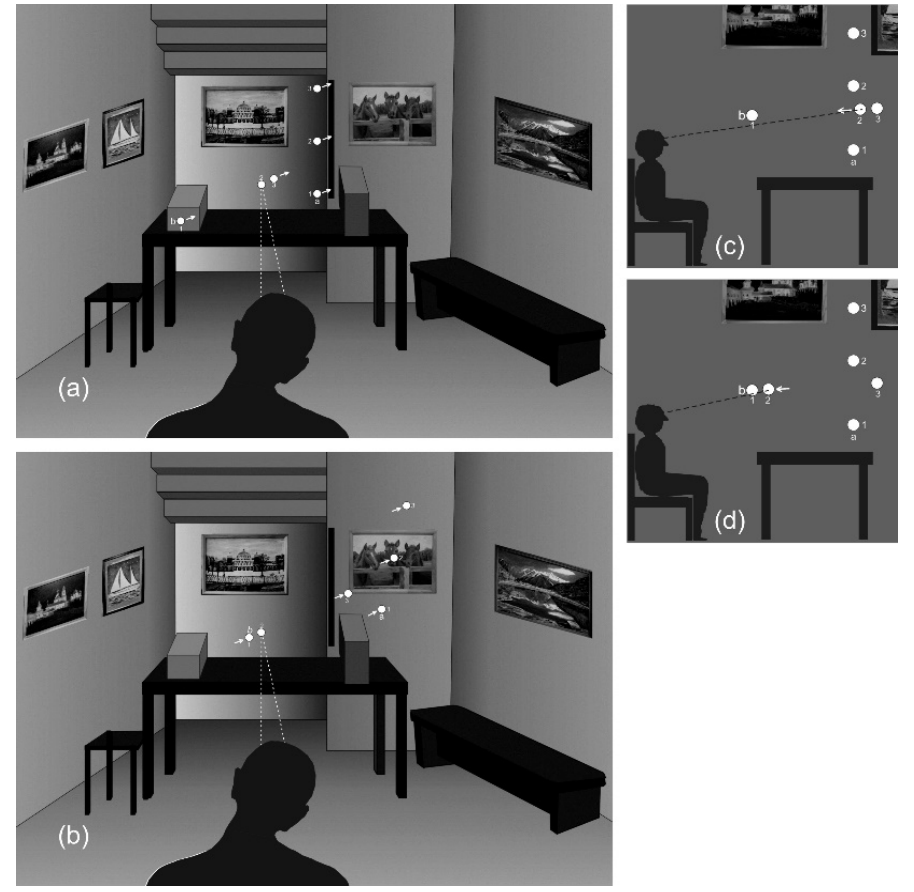

Figure 2. The illusion of the "shift of the IVS in depth" when tracking the point b2, which moves smoothly towards the observer. The subject's head is tilted to the shoulder (position 10). (a) The visual field of the subject in the optimal development phase of rA. The subject tracks the smoothly approaching point b2; (b) the apparent movement of an objectively stationary configuration of luminous space markers in the direction from the subject with apparent immobility of the actually moving b2 point; (c), (d) the actual location of the MRS in the initial (c) and final (d) tracking phases of the moving point (side view)

The subjects described more complex impressions with respect to the apparent change in the size of the reference points. In particular, the apparent size sometimes changed to varying degrees for the fixation point and the rest of the configuration points. In more details this feature of the phenomenon of illusion in these experiments has not specified.

The oblique position of the head by about $25^{\circ}$ in some way influenced the features of the illusion, if we compare it with the illusion in the normal (00) position of the head-rA. When the head was tilted, the orientation of the vertically oriented block of points a (1-3) as already noted above apparently changed. The direction of its inclination depended on the direction of the head inclination-it always occurred in the same direction, as the head tilt was. In the normal position of the head, the block of points a (1-3) remained vertical.

An interesting moment of the experiment was when the subject sometimes paid attention to the fact that the point b3, which was moving away from observer, went outside the room and was apparently located behind the virtual back wall of the room.

All the main effects of these two illusions were recorded by all eight subjects who participated in the experiments. In these experiments, some other essential features of the rA illusion were investigated in more detail.

\section{The residual illusion phenomenon}

Here we describe another important and for some properties paradoxical phenomenon, studied in detail in these experiments. It has already been noted that the illusion caused by smooth or saccadic eye movements lasts not only from the moment of the movement start for the entire duration of $\mathrm{rA}$, but also observed for some time after the 
complete attenuation of $\mathrm{rA} \mathrm{[3].} \mathrm{Already} \mathrm{being} \mathrm{in} \mathrm{complete} \mathrm{darkness,} \mathrm{the}$ subject reports that the new position of the MRS remains unchanged for at least 15 to 30 seconds. After this time, the reference points sometimes smoothly displacing, sometimes without visible movement return to their "normal" position in real space. We called this phenomenon "residual illusion" (RI).

The residual illusion lasts much longer than the rA itself which caused it. It was found that a particularly prolonged RI sometimes was observed when fixation point moved in depth (as in the experiment in Figure 2). In some cases, it lasted up to 1.5 minutes. In the present work, no statistical estimate was made of the inter- and intra-individual variation of the RI duration. Its scatter from experiment to experiment was clearly significant. Here it is important the fact that the RI has been reported by the all without exception subjects and the spreading of its duration was in the range from 15 to 90 seconds.

Figure 3 shows schematically the time development of rA brightness and RI described by an observer in one of the experiments given in section 1 .

The final RI phase, as described by all subjects, was especially clearly recorded by changing the apparent slope of the point block $b$ (1-3) which gradually returned during this period (and sometimes without visible movement) to its original horizontal position, changing its angle of inclination by about $20^{\circ}$.

During the observing of RI, it is possible to carry out of a set of additional actions, which we describe with the example of the "vertical shift IVS" illusion, shown in Figure 1. As already noted, similar an illusion can also be caused by a saccadic eye movement when changing fixation for instance from point a3 to point a1. In this case the entire configuration of MRS jumps up just as shown in Figure $1 \mathrm{~b}$.

Below some characteristic phenomena observed in the residual illusion phase described.

I. RI can be interrupted at any time if during the period of its observing eyes perform the movement reversed to that caused the illusion itself. In the experiment presented in Figure 1 the saccade can be such movement from bottom to top-from a2 back to a3 (Figure 1d) already in full darkness. In this moment the illusion disappears at once and all MRS instantly return to its "normal" position in space (remember that before that the eyes smoothly moved on the background of rA from top to bottom).

II. However, if in the RI phase a new eye movement (for example, a saccade) occurs along another coordinate axis of the 3-D space, a new combined illusion arises already in the absence of rA. So, if using the example of the experiment in Figure 1, the subject's eyes make a leap from point a 2 to point b1 (the relative position of the points can be seen in Figure 1a and 1d) both the deep and the horizontal components will be added to the vertical illusion: all the MRS, which are currently located at the top under the ceiling, will also move in depth and to the right. And this new illusion will last as RI all the time allotted to it, and then all the MRS will return to the normal position, sometimes smoothly shifting in all three coordinates.

III. At the same time, if during period $\mathrm{rA}$ point a2 passes only half the way from top to bottom, causing an illusion of half degree and already in RI phase continues moving down to complete its full path, the illusion will immediately disappear. The same will happen if from this intermediate position a 2 the subject makes saccade down to point a1, as if completing the procedure of shifting the point a2 down. If the subject makes such an additional saccade against the background of
$\mathrm{rA}$, the illusion of a vertical shift of the IVS will manifest itself in full. (Smooth eye movement in this case will be replaced by a saccade).

IV. If in the RI phase, when the subject continues to fix point a2 already in the dark, the experimenter begins to manually smoothly move the additional luminous point near point a3, which is physically located at the level of his head, the subject exclaims with surprise how the experimenter managed to reach point a 3 located below the ceiling (as seen in Figure 1b). A new test point performs voluntary movements under the ceiling in the subject's altered visual space, without affecting the RI itself until its completion.

Disturbance of the motor spatial orientation of the subject during the RI period will manifest itself in an obvious way if by the direction of the experimenter a subject fixing a 2 point tries to touch point a 3 with a pointer with a luminous tip. He pokes his pointer in the ceiling area of the real experimental room, where physically there is no luminous point.

$\mathrm{V}$. Here we describe in some way a limiting case of manifestation of RI in the complete absence of MRS from the flash moment until the final RI period. Under the conditions of the "dark mode" experiment, the subject before the flash in complete darkness fixed an imaginary point a3, and after the flash, turned his gaze to an imaginary point a1. And only at a pre-calculated time in the period of the final part of the RI MRS were switched on. Experiment has shown that the subjects still saw the illusory arrangement of the entire MRS configuration in space and then its smooth (sometimes "instant") return to the normal position. This moment of the experiment "in the dark mode" is shown schematically in Figure 3 (Zone $\mathrm{F}$ to the right of the arrow).

VI. A bright impression on the subject is made by the monocular observation of RI, when the subject first sees the illusion of a vertical IVS shift with one eye, and in the RI phase changes eyes, closing the one that was open in the flash moment and opening the previously closed one. To his surprise, the subject fixes the effect of "transferring" the illusion from an eye to eye, observing the same RI in its final phase when block b (1-3) smoothly changes its orientation, returning to the horizontal position.

\section{Illusions of rA under head movements}

In the experiments described here, subjects in connection with special conditions (tracking a moving point when the head and/or image are tilted) were initially specifically instructed to make movements only with their eyes when the head was stationary. This condition was based on the idea that head movements under certain conditions can

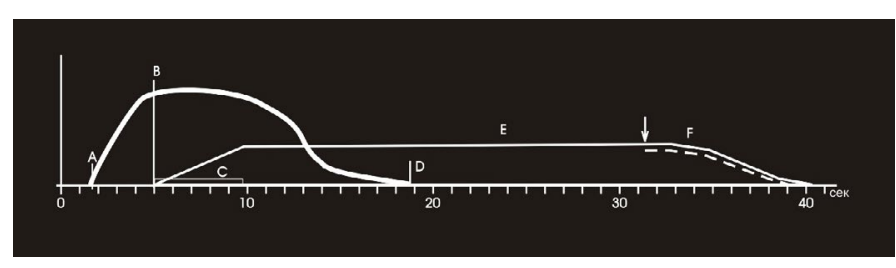

Figure 3. Schematic representation of the $\mathrm{rA}$ development and the illusion in time under smooth movement of the eyes, tracking the moving point a2 (shown in Fig. 1). The ordinate axis is the conditional display of the time-varying rA brightness. The designations in the figure: point 0 - flash point; $\mathrm{A}$ - the moment of the initial development of the $\mathrm{rA}$; B - the beginning of the smooth movement of a2; $\mathrm{C}$ zone - the period of smooth eye movements tracking the moving point; E - conditional line of development of the initial illusion phase, as well as residual illusion, which lasts in this case for more than 20 seconds after complete attenuation of the rA (point $\mathrm{D}$ ); the arrow is the moment when the MRS were turned on in the "dark version" of the experiment described above in section V; zone F - the final period of RI 
significantly affect all the main phenomena that manifested themselves in experiments with isolated eye movements.

Reality has significantly changed these expectations. A wide series of experiments were carried out in which the change of the fixation point was performed only with the help of fast or slow head movements without changing the position of the eyes in the orbits. The subject monitored the latter condition independently and gained experience in its successful implementation. The results of these experiments showed that all the phenomena of $\mathrm{rA}$ illusions are fully preserved under head movements.

The angle of inclination of the head in the mediate plane in experiments with tracking the vertically moving point a 2 or turning the head when looking from point a1 to point $\mathrm{b} 2$ was $15^{\circ}-25^{\circ}$. In a more complex combination, when looking from point b3 to point b1, it was $5^{\circ}-7^{\circ}$. With all such movements of the head, rA remained unchanged, while the MRS performed the same "illusory" shifts in space as with isolated movements of the eyes. At the same time, the residual illusion fully manifested itself.

After the completion of the head movement, the subjects sometimes claimed that their head seemed not to make a movement and was still oriented in the same way as before the flash. Such a false sensation of the head state lasted for some subjects the entire period of residual illusion.

One of the modifications of the experiments with the head movements is shown in Figure 4. In these experiments, point a2 was attached to the end of the plastic bar. The bar was firmly fixed on the subject's headdress. Before the flash, the subject focused his head so that point a 2 was in the subject's field of view near point a 3 at a distance of about $70 \mathrm{~cm}$ from the eyes. After the flash, the subject smoothly changed the inclination of the head, so that the point a2 moved to a fixed point a1.

The subject strictly fixed the point a 2 all the time the head moved. In different series of experiences, the head was oriented exactly vertically or was tilted to the shoulder. In these very unusual conditions, the effect of the vertical shift illusion of IVS was completely preserved: the subject saw the moving point a 2 as stationary. All the other points-MRS smoothly shifted upwards with the previously noted slope of the block b (1-3). RI manifested itself as in all previous experiments (Figure 4).

\section{The illusion of the visual vertical}

When conducting a series of experiments with oblique rA, one more illusion was recorded, expanding the range of influence of the entire visual scene afterimage on visual effects. This is about the illusion of deviation of the visual vertical. The question of the visual perception of the gravitational vertical is important because it defines one of the main axes in the IMSS and IVS coordinate system. Nevertheless, the possibility of its mismatch in these two models is well known $[4,5]$.

In our experiments the illusion of the visual vertical deviation was detected at the moment when the subject, tilting the rA with the movement of the head drew attention to the fact that the vertically exposed block a (1-3) against the background of the oblique rA changes its apparent orientation, deviating to the side opposite to the rA tilt. This illusion manifested itself in both the direct and tilted final position of the head (in situations 01 or 11).

The quantitative characteristics of the illusion as well as the accompanying other visual effects will be described elsewhere. The main goal of this series of experiments was to find out the existence of residual component of this illusion in these conditions. A clear answer has been received to this question: this illusion also had a residual component. The apparent slope of the vertical remained after the complete attenuation of rA for another 15-30 or more seconds after which the block of dots a (1-3) most often smoothly turning around (less commonly without visible movement) returned to its normal upright position.

This allowed the subject already in the RI phase to accurately set the block of points a (1-3), leaning against the background rA, with the help of a simple mechanical device in a subjectively vertical position. This fact could be recorded using photography.

In Figure 5, we see fragments of a similar experiment.

\section{Gaze direction and head position in the RI phase}

When the experimenter paid the subject's attention to the impression of where his gaze was directed or in what position his head was oriented, the answer was always erroneous. For instance, when the subject changed his gaze from top to bottom, for example, from point a 3 to point a1, his gaze according to the subject's opinion was still directed upwards, although physically the eyes were already oriented horizontally and the vertical angle of gaze has changed by more than $20^{\circ}$ (Figure 1d). The same thing happened when tracking a moving point a2. This illusory direction of gaze was maintained in the phase of residual illusion until its completion.
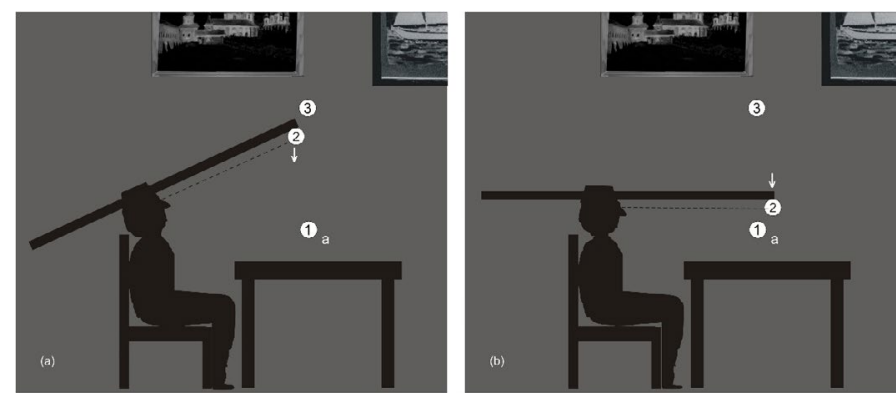

Figure 4. Modification of the experiment in Fig. 1, in which the movement of point a2 against the background of rA was provided by the movement of the subject's head, to whose headgear a fixation point was attached to a special rod at $70 \mathrm{sm}$ from eyes. (a) - The position of the head and the fixation point before the flash; (b) - the final position of the head and point $\mathrm{a} 2$ after turning the head against the background of $\mathrm{rA}$

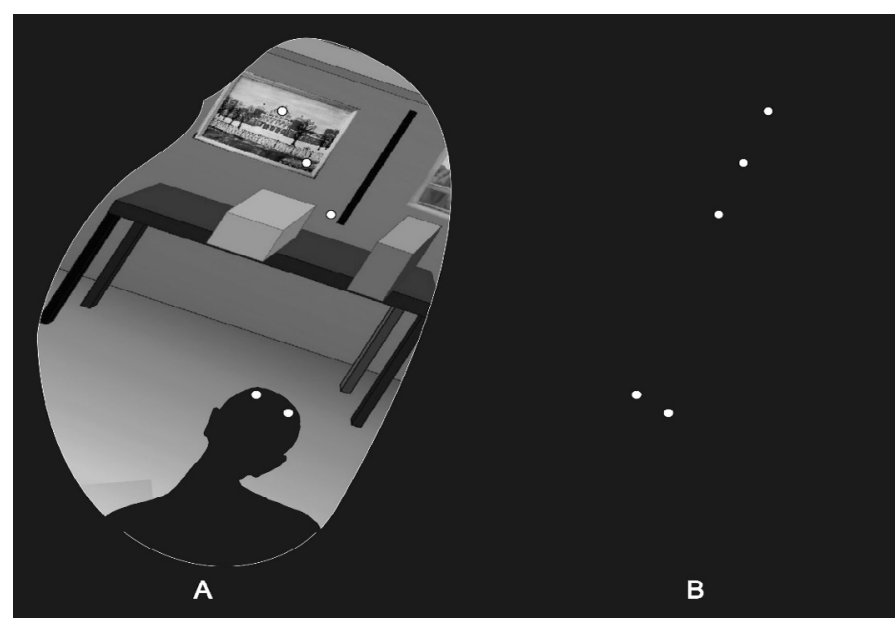

Figure 5. A- a fragment of the subject's visual field and the entire experimental situation in the mode of fixing the angle of inclination of the visual vertical against the background of rA; B - photo fixation of the actual orientation of block a (1-3) after the subject has set the subjective vertical already in the phase of the residual illusion 
It can be said that even more impressive was the subject's erroneous feeling about the position of his own head when the subject changed the direction of his gaze only by turning the head, keeping the position of the eyes in the orbits unchanged. Sometimes for the entire time of RI he had the false impression that the head was still oriented upwards (as shown, for example, in Figure 4A) or straight ahead when changing the gaze from point a1 to point $\mathrm{b} 2$.

An experienced observer sometimes described the dynamics of changing sensations about the position of the head in the residual illusion phase. From confidence that the head remained oriented upward, the subject proceeded to experience a loss of sensation in general about the position of the head (up to a loss of the neck muscles feeling), and finally it became clear to him that the head was straight oriented and the gaze was strictly directed to the lower point. We note a complete analogy in the dynamics of experiences when changing the point of fixation with the saccade: from false confidence through loss of understanding where the gaze is directed and finally to full confidence that the gaze is directed to the previously specified final point of fixation.

In experiments with changing the point of fixation with a saccade, an inexperienced subject sometimes reported that he was unable to follow the indication of changing the direction of gaze: he still looks in the same direction at the same stationary point, although he saw that all others points shifted for some reason. Sometimes the observer reported a clear painful discomfort from the contradiction between the sensations of gaze direction and the eye position in orbits.

\section{Discussion}

In the scope of the current article, it is not possible to discuss and adequately interpret meaning all the phenomena observed in the experiments and described here. We will dwell here only on a certain hypothetical fundamental interpretation of the points that require discussion in the first place, at least in the preliminary and most general "sense".

Unlike experiments with violation of one way or another of the operation of the eye-motor executive system, aimed in one way or another to clarify the principles of operation of the visual field stability mechanisms, in experiments with $\mathrm{rA}$, the oculomotor subsystem remains completely intact and adequately responds to all signals from the level of eye movement control (we add that the same can be said about the work of the neck muscles) [6-13].

In our experiments, the eyes accurately change their position and, in accordance with the control theory in complex systems, should send a return signal to the upper levels about performance. But at the same time, the system in the visual channel itself, which is also completely functional, does not receive signals about the movement of the entire retinal image of the visual scene to a new position, nor about the speed of the movement.

And this turns out to be enough so that against the background of $\mathrm{rA}$, the mechanisms of the visual system responsible for the visual field stability produce a completely "inadequate" reaction. "Inadequacy" is expressed primarily in the fact that the signals about ideally performed actions of the oculomotor system are completely ignored. Moreover, experiments with head movements show that the "more powerful" signals of the neck muscular system, which are responsible for head movement, can also be completely ignored.

This happens not only against the background of the unchanged rA, but continues for tens of seconds already in complete darkness in the phase of residual illusion. And all this time, the IVS remains completely out of alignment with the internal model of the surrounding space (IMSS) in all three spatial coordinates. And new eye-leaps in the dark can still be ignored and lead to a visible modification of the ongoing illusion (section 3.II).

A seditious question arises: does the mechanism of the visual field (IVS) stability need efferent signals about eye movements in general? After all, when an eye leaps under normal conditions, the system has access to all information in the visual channel and on the magnitude of the optical shift of the retinal image that actually occurred and on its speed.

So why it may not be enough, and why the system should first rely on signals from the executive oculomotor subsystem (as many theories of stability mechanisms suggest) instead of using extremely informative events occurring in the visual stream itself primarily for an operation of the mechanism of the visual field stability? [13]. Here we would like to justify and propose for discussion a hypothesis about purely afferent mechanisms of the IVS stability, adding preliminarily an additional analysis of the phenomena observed in the experiments described here.

In this regard, we turn to the very phenomenon of $\mathrm{rA}$ as the afterimage of the entire visual scene. Its main feature is that in perception it seems to the observer to be completely indistinguishable up to a certain moment from the real three-dimensional visual field observed in ordinary visual conditions.

The stereopsis mechanism formed this image from two "frozen" retinal images, and its synthesis also did not require any "auxiliary" eye movements-they simply cannot bring any fine tuning for two frozen images. Note that in some experiments this ideally formed 3-D view of the scene appears before the observer, who first saw it only after the flash, because one was initially placed in a dark room and went through a period of dark adaptation, seeing nothing but some luminous points.

A subject observes this "ideal" image of the visual scene already in complete darkness. And if, immediately after flesh, a subject turns his head horizontally by $90^{\circ}$, he will see the same 3-D scene, which is in no way connected with a real physical environment. Thus, in these experiments it is obvious that the subject is observing his IVS, or it may be possibly to say, "the inner visual room". That is why we are from the very beginning talking about the internal 3-D visual space. And this room is perfectly formed without the participation of any auxiliary eye movements. (Meanwhile, there is a generally accepted view that the role of eye movements in the mechanisms of stereopsis is very large).

An even more significant effect is observed in position 11, when immediately after a flash with the head tilt to the shoulder, the subject sees an inclined field in which all spatial relations between all elements of the scene are perfectly preserved.

This 3-D field is formed not only without the participation of any auxiliary eye movements (except for their turning by the very not simple way around the gaze axis when the head is tilting to the shoulder [14-16]. In its inclined position, it reproduces the visual scene in all its details, is completely divorced from any morphological links with a mosaic of photoreceptors. Indeed, at this moment two frozen, still "luminous" retinal images of the same scene are presented in their original, normally oriented position in relation to the retinal meridians.

If during the period of a good formed $\mathrm{rA}$, which can last about 10 sec the subject smoothly returns his head to the normal position he will see a three-dimensional scene returning to its normal orientation. But now it becomes "obvious" that the coincidence of three equally 
oriented objects - two retinal images and 3-D view of the scene - is just a "coincidence": the head tilting to the shoulder this seemingly hard link will immediately destroy again.

In this new arrangement of the oblique view of scene, shifting the focus of attention within the field again does not encounter any difficulty in the subject: gaze control mechanisms successfully cope with this, although no geometric correspondence with the retinal receptor layers already exist.

We must also recognize now that the IVS as an internal model isomorphic to the external 3-D scene acts as a fragment of a more general internal egocentric model isomorphic to the real space of the current environment with the subject at the starting point of the internal coordinate system. Since when one turns the head in normal vision, new parts of the scene that come into view will fit into the updated IVS along with the others that will disappear. The updated IVS as a product of the visual system will emerge as before as the visualized ("lightened") fragment of IMSS.

We also note that in the experiment shown in Figure 1, when the eyes move in a secondary position, and in the experiment in Figure 2 in a complex "tertiary" position, the cortical transformation of curved retinal coordinates, assumed in these cases, should also is carried out without any participation of eye movements [17].

For our consideration, the head-rA position 10 is no less informative. As shown in Figure 2, the illusion of an "IVS shift in depth" is manifested against the background of a normally oriented $\mathrm{rA}$ when the head is tilted. Under these conditions, the subject observes the same 3-D scene without any manifestation of diplopia and without visible distortion of the spatial relations between the elements of the scene.

Meanwhile, the geometrical pattern on two receptor fields under these conditions is dramatically changing. Figure 6 schematically shows how the position of the scene elements changes when the head is tilted to the shoulder using the example of two marker points of the scene.

Suppose that at the normal position of the head two points 'a' and ' $b$ ' are projected on the median horizontal retinal meridians and their disparity relations can be used to determine their depth position (Figure $6 \mathrm{~A}$ ). In this position there is a temptation because of the simplicity of this picture to tie the disparity of these points (as well as all other points of the field) to the morphological structure of the two photoreceptor layers (to the retinal corresponding points).

However, when the head is tilted to the shoulder, the projections of these two points due to different distances to them will not only move to different meridians (Figure 6B), but these meridians may be in different angular positions. At the same time, all points of space located in depth between points $\mathrm{a}^{\star}$ and $\mathrm{b}^{\star}$, previously projected on the median horizontal meridian, will occupy a whole zone between meridians $\mathrm{a}^{*}$ and $\mathrm{b}^{*}$ and these zones may have different vertical size.

Every zone is still horizontal to the scene's plane to be oriented at a significant angle to the retinal main meridian. In turn, each meridian is leaned at an angle smaller than the angle of the head inclination by the weakly tied to the head tilt angle of residual torsion especially in the dark $[14,16]$.

Besides the horizontal component of the interpupillary base (the horizontal distance between the eye pupils), which is so important for the binocular synthesis of IVS, also change, for example, when the head is tilted $45^{\circ}$ almost 1.5 times.

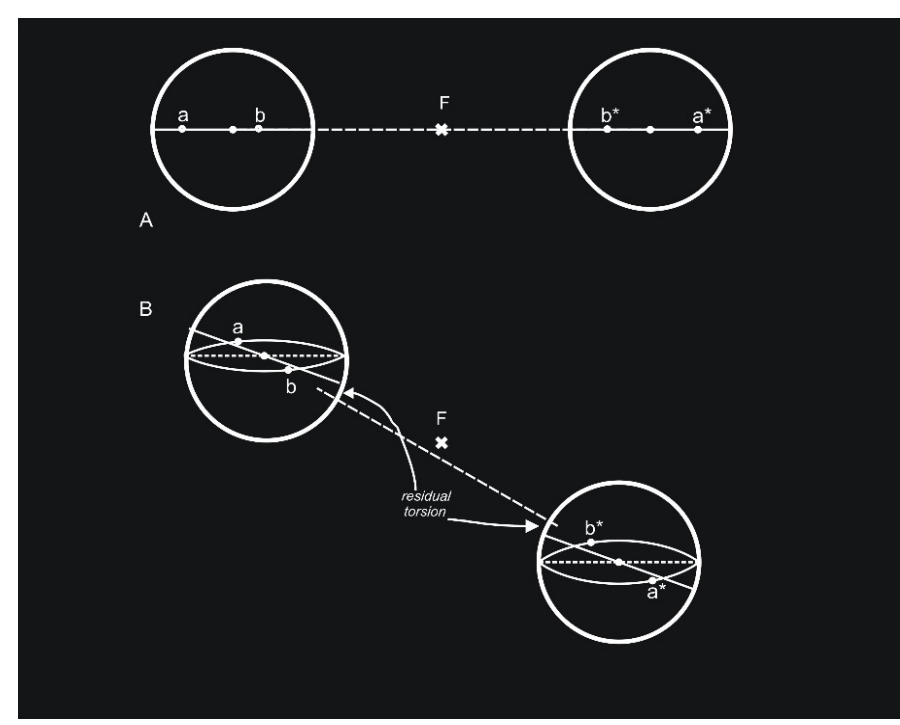

Figure 6. Schematic representation of the stimulus situation on the photoreceptor layers of two eyes when the head is tilted to the right shoulder. All explanations are in the text. (A similar scheme has already been discussed earlier [14]

To say that in this case some morphological components of correspondence and disparity may be maintained or corrected in some regular way hardly makes sense.

In the experiments with $\mathrm{rA}$, this situation is aggravated by two more circumstances: (1) eye movements in these difficult conditions can in no way participate in any correction of the "relative positioning" of two retinal images, while (2) the point that the subject fixes in complete darkness up to the flash moment, it may be in no way connected with the elements of the scene (which was the case in most of the experiments). In addition, under these conditions, the fixation reflex cannot function adequately, because the fovea centralis zone is practically blind in the case of the rA rod view.

This means that the binding of mutually corresponding local areas of two retinal images can be realized in a block of stereopsis mechanisms only on the basis of their geometric visual features inside the images.

Ultimately, experiments with oblique rA convince us that the fusion mechanism, as an instrument of stereopsis, is capable of forming a fused 3-D IVS without any auxiliary eye movements only on the basis of a comparison of the geometry of two inner images. Within these images purely visual geometric projective relations of correspondence and disparity are formed, which are not tied in any way to the morphological structure of two mosaics of photoreceptors.

A certain confirmation of these ideas can be found already in experiments with a short (less than $200 \mathrm{~ms}$ ) exposure time of the visual scene, in experiments on the formation of the visual field under conditions of pseudoscopic vision, under conditions of observation of the underwater illusion [18-20].

Thus, the whole complex of phenomena and illusions in the experiments with $\mathrm{rA}$ can convince us of the need to revise the functional role of eye movements in the process of forming a constant 3-D visual field. And we are ready to take the courage and to express as a hypothesis, the "Uttermost Afferent Theory of Controlled Projection" (UATCP).

To substantiate and refine this hypothesis, we now turn to the paradoxical phenomenon of residual illusion (RI), described here in 
fact for three types of illusions. If against the background of $\mathrm{rA}$, each of the illusions can be "explained" by the presence of the background of straight or inclined rA as a kind of "reference system", then in the dark under normal signal processing of all efferent mechanisms need to explain the two sides of this phenomenon.

First, with respect to which coordinate system the observer continues to see and report so long the fact of illusion? Secondly, what does not allow the system in complete darkness at once to return to the "correct" interpretation of the spatial situation in the oculomotor, in the cervical-muscular or in the vestibular systems?

In response to the first question, we must assume that such a system can be the already mentioned the internal model of the surrounding space (IMSS), in relation to which the internal visual 3-D field (IVS or may be IVR) turned out to be mismatched either by position or by orientation (or perhaps also by scale), which is interpreted in the mind of the subject as an illusion. This mismatch extends to assessing the direction of gaze, the position of the head, and the orientation of the visual vertical.

The "culprit" of the second peculiarity of the RI, namely its considerable duration already in complete darkness with complete absence of visual stimulation, according to our hypothesis, are "screen invariance mechanisms" (SIM) responsible for the coordinated work of all levels of the visual system, providing a stable IVS. The need to recognize the existence of such screen mechanisms was expressed earlier [1].

These mechanisms are "screen" or may be "field", "iconic", "nonobject" since their work does not depend on the object content of the visual scene and the object structuring of the visual field. These mechanisms have an unexpectedly high functional capacity, sufficient to hold the perception of the MRS position, and the gaze direction, and the head orientation, and the orientation of the visual vertical in a distorted relative to their real state for tens of seconds in the complete dark. Namely, inadequate conditions in the visual channel (and not in the efferent channels) in which the visual system was placed, forced SIM to "persist" for so long and to keep all these components of perception in a distorted state.

As for the illusion of the eye and head movements, then "from the point of view" of the visual system which has not received an adequate signal in the visual channel about the displacement of the entire field as a result of the movement of the eyes, it can only be interpreted as an external event, as a real movement of isolated external objects-MRS. It is fixed and thus already in the dark for the visual system or for the mind there is no reason to accept this event as an illusion.

But things are deeper. The illusion arises also in the absence of MRS and refers to the transformation of the coordinate grid of the entire 3-D space. So, it is a product of the functioning of the fundamental intermodal mechanisms and not only the visual system. Since the illusion eventually disappears, it means that from a more general position of the body orientation in space it is still a kind of violation of internal relations that must be eliminated. This is what happens in the end.

To the hypothesis of SIM we should also add ideas about the hypothetical level of the system at which they realize their function. Earlier we called this level "constant visual screen" (CVS); here we constantly mention IVS. It can be assumed that these are really two different "formations": at the CVS level, the object structuring of the field may not yet be realized, while the IVS is already object-structured (object-specific).

In this regard, the level of IVS can be considered as an analogue of Julesz's "cyclopean retina” [21].

The introduction of such a level suggests that the visual perception itself is "vision" not of the retinal image, but of the product of the retinal image projection through hypothetical SIM and object structuring mechanisms and stereopsis on IVS. And the sloped 3-D rA, as already noted above, is the first evidence of that. Now, based on all the above, we detail the hypothesis UATCP and decipher it terminologically.

We called this hypothesis "Uttermost" because it boldly proclaims that the efferent signals of the oculomotor system do not participate in any phase of a stable visual field formation. Therefore, this theory is called "Afferent", since only signals in a purely visual channel participate by screen invariance mechanisms" (SIM) in the formation of a stable IVS. These hypothetical SIM carry out the "controlled projection" of the retinal image onto a certain upper level of the visual system, which has been called IVS here (It is possible this can be called also IVR-internal visual room in view of its always inherent spatial restriction).

"Controlled projection" is carried out considering all new projection relations that have arisen on the two fields of photoreceptors as a result of eye movement. It is "Controlled", since for its execution it is necessary to have also an "enabling" signal from the control centre for eye and head movements.

Noteworthy are the differences in visible effects with horizontal or vertical on the one hand and rotational around the gaze axis on the other movements of the head. In the latter case, as already noted, It appears in experiments with $\mathrm{rA}$ as a visible rotation and tilt of the entire visual field with all its 3-D object-spatial content, even if there are details of the observer's own body in this field (for example, palms or outstretched arms). This IVS rotation occurs in the opposite direction with respect to the optical turn of images on the photoreceptor field in normal vision.

The reason for the difference between these two types of movement remains unclear, and only it is possible to assume that the rotation the eyes and the head around the gaze axis as opposed to horizontal or vertical rotation the eyes and the head is worked out by internal SIM in a different way.

If we accept everything stated here as a working look useful for planning further research then we have to admit the only purpose of eye movements, according also to classical ideas, is to move the "fovea gaze" to the planned point (local zone) of the visual scene to "re-write" the selected zone with maximum details [5,22]. And the strategy of the whole complex of such involuntary movements occurring under normal conditions 2-4 times per second is to perform this function for the maximum number of zones of the visual scene [22].

These eye movements are necessary for the visual system due to the extremely small size of the high-resolution area ("fovea gaze") and therefore these movements are of practical importance for the work of the control centers of the entire nervous system. If, according to the literature, the main working area of the visual field ("nearest periphery") is $30^{\circ}$ in all directions from the 'fovea centralis' and the size of the highresolution fovea zone is $5^{\circ}$, then the ratio of these two areas is close to $1 / 150$ and it is possible that this is a markedly exaggerated estimate.

Since we consider IVS as an "illuminated" fragment of the IMSS, the UATCP hypothesis can be further strengthened if to assume that 
all decisions of the eye movement by control centre are made on the basis of 3-D IVS geometry and coordinate relations between target (and related objects) of current task inside IVS model, not retinal images. But if the gaze translates beyond the limits of the current visual field with additional head turning, the whole complex of movements is regulated based on the geometrical parameters and the object content of the IMSS zone nearest to the visual field. If this zone has already been in the visual field at least once, then its object filling and geometrical relations inside this zone have been recorded in the IMSS state.

For various reasons, the visual system may not even be very "concerned" with the absolutely accurate execution of the motor task, since the actual shifts of the optical situation on the retinas are determined with high accuracy in the visual channel and, if necessary, can be corrected by additional eye movements [22].

Now we will pay attention to the fact that during eye movements besides the frozen $\mathrm{rA}$ there is also a moving MRS configuration in the visual field. But signals about its movements are not significant for stability mechanisms with the presence of $\mathrm{rA}$. But with a small or poor afterimage (pA), these signals are enough for adequate functioning of these mechanisms. As a result, the pA frozen on the retina is projected into a new IVS area, making a visible movement in sync with eye movements-a violation of the relationship between IVS and IMSS does not occur. In this regard, it may be productive to consider $\mathrm{pA}$ as a model of a single object or a figure, and $\mathrm{rA}$ as a model of the background or "ground" and the concept of Gestalt psychology about the deep functional figure-ground difference between an object and background acquires in some way another experimental support.

This is supported by the fact of the illusion of a visual vertical, when the power of the oblique $\mathrm{rA}$ is enough to disrupt the fundamental reflex of visual perception of the gravitational vertical. In complete darkness and with a tilted head, a person is usually able to almost perfectly expose the visual vertical. But in the presence of the oblique $\mathrm{rA}$, even with a normal oriented head, the subject makes a systematic error in the visual assessment of the vertical. It is surprising only that the violation of the visual vertical perception still remains in complete darkness in the RI phase, lasting for tens of seconds. The return of the vertical to the normal position reported by the subject as an "obvious" smooth return of the test block of points to normal orientation can be a very informative fact if it will be found out in further experiments that it is not accompanied by any "substantial" adequate eye movements.

It is difficult to talk about the possibility of convincingly interpreting based on UATCP all the observed visual phenomena accumulated over a long history $[5,11]$. A general review of these results from the perspective of UATCP may require the introduction of some efferent component into the general flow of control signals. And the bold hypothesis proposed here now is more likely to raise more new questions than to answer for all existing ones. However, one can say that the phenomena found in the experiments force us to take a fresh look at the role of a purely visual signaling system in setting many functionally important parameters of the body.

Thus, we would like to view the visual system primarily as a builder of "theatrical scenery" (and not as a measuring device of a spatial relationship), surrounding the "actor" (he is also an observer) to make one's stage activity in space (including also a measuring procedures based, in particular, on the IVS perceptual analysis [23]. Therefore, in front of one's eyes, the well-named "visual scene" is located, the internal model of which is IVS or IVR or IVF-"internal visual space or room or field", which can be considered synonymous.

A stable figure structured IVR is the only purely visual product of all the visual system mechanisms. When rendered outwardly by the mysterious mechanisms of visual perception, it becomes that "external" visual field, which in normal conditions is indistinguishable from the visual scene of physical space. And the methodology of experiments with $\mathrm{rA}$, which violates perfectly organized links between the external optical flow and the internal information flows in the visual system, only reveals the peculiarities of their mutually agreed operation mechanisms.

\section{Competing interests}

The author declares that he has no competing interests.

\section{Acknowledgements}

The author is sincerely grateful to Dr. D. Nikolaev and Dr. G. Rozhkova for the organizational support of this work, as well as A. Belokopytov for the permanent technical assistance.

\section{Funding}

The author(s) received no financial support for the research, authorship, and/or publication of this article.

\section{References}

1. Zenkin GM, Petrov AP (1976) Transformation of the afterimage by observer' movements and visual field constancy mechanisms. Human Physiol 2: 925-931.

2. Zenkin GM, Petrov AP (2015) Transformation of the visual afterimage under subject's eye and body movements and the visual field constancy mechanisms. Perception 44: 973-985. [Crossref]

3. Zenkin GM (2017) Rich afterimage illusion and inflow on-screen mechanisms fo visual field constancy and visual orientation in space. New Front Ophthalmol 3: 1-8.

4. Brandt T, Dichgans J, Held R (1973) Optokinesis affects body posture and the subjective visual vertical Pflugers Archiv 339: 97.

5. Belopolsky VI (2007) The gaze in man. Mechanisms, models, functions. Institute of Psychology, Moscow, Russian Academy of Sciences pp. 416.

6. Hoist E von, Mittelstaedt H (1950) Das reafferenzprinzip (Wechselwirkungen zwischen zentralnerven-system und peripherie) Naturwissenchaften 37: 464-476.

7. Hoist E von (1954) Relations between the central nervous system and the peripheral organs. Brit J Anim Behav 2: 89-94.

8. Sperry RW (1943) Effect of 180 degrees rotation of the retinal field on visuamotor coordination. J Exp Zool 92: 263-279.

9. Sperry RW (1950) Neural basis of the spontaneous optokinetic response produced by vision inversion. J Comp Physlol Psychol 43: 482-489. [Crossref]

10. Stevens JK, Emerson RC, Gerstein GL, Kallos T, Neufeld GR, et al. (1976) Paralysis of the awake human: visual perceptions. Vision Res 16: 93-98. [Crossref]

11. Matin L (1976) A possible hybrid mechanism for modification of visual direction associated with eye movements - the paralyzed eye experiment reconsidered. Perception 5: 233-239. [Crossref]

12. Matin L, Picoult E, Stevens JK, Edwards MW, Young D, et al. (1982) Oculoparalytic illusion: visual-field dependent spatial mislocalizations by humans partially paralyzed with curare. Science 216: 198-201. [Crossref]

13. Barabanshchikov VA, Belopolsky VI (2008) Stability of the visible world. Moscow: Institute of Psychology. Russian Academy of Sciences. 303 pp.

14. Petrov AP, Zenkin GM (1973) Torsional eye movements and constancy of the visual field. Vision Res 13: 2465-2477. [Crossref]

15. Galoyan VR, Zenkin GM, Petrov AP (1976a) The investigations of the torsiona eye movements in man. I. Some features of torsion movements when tilting head to shoulder. Biophysics 22: 570-577. 
16. Galoyan VR, Zenkin GM, Petrov AP (1976b) The Investigations of the torsional eye movements in man. II. The slow phase of the torsion. Biophysics 22: 1081-1086. [Crossref]

17. Crawford JD, Henriques DYP,Vilis T (2000) Curvature of Visual Space under Vertical Eye Rotation: Implications for Spatial Vision and visuomotor Control. J Neurosci 20: 2360-2368

18. Ogle K (1962) The Optical Space Sense. In: Davson H (Eds) Visual Optics and the Optical Space Sense. Vol. 4, Academic Press, pp. 211-417.

19. Stolin VV (1976) Study of generation of the visual spatial image. In: Leontiev AN (Eds) Perception and action. Moscow University Press, Moscow, pp. 101-208.
20. Petrov AP, Zenkin GM (1976) The retinal images as sources of information about the eye positions in the orbits and binocular mechanisms of the visual space formation. Human Physiol 2: 932-939.

21. Julesz B (1971) Foundations of Cyclopean Perception. University of Chicago Press, Chicago.

22. Yarbus AL (1967) Eye movements and vision. Springer, Boston, USA.

23. Zenkin GM,Petrov AP (1987) Functional organization of the visual process and the gestalt principle. In: Velikhov EP, Chernavsky AV (Eds) Intellectual processes and their modeling Nauka, Moscow, Russia, pp.264-292.

Copyright: $(02019$ Zenkin GM. This is an open-access article distributed under the terms of the Creative Commons Attribution License, which permits unrestricted use, distribution, and reproduction in any medium, provided the original author and source are credited. 